\title{
BER and MSE Performance of MIMO-OFDM System using Channel Estimation Technique
}

\author{
Kratika Sharma \\ M.E Scholar \\ Department of Electronics \& Communication \\ UIT-RGPV, Bhopal, India
}

\author{
Anubhuti Khare, $\mathrm{PhD}$ \\ Professor \\ Department of Electronics \& Communication \\ UIT-RGPV, Bhopal, India
}

\begin{abstract}
Next generation mobile systems will use multiple antennas at the transmitter and receiver to achieve higher capacity and diversity gain at high speeds. By transmitting through multiple transmitting and receiving antennas, multiple wireless data pipes are created. A transmitted signal while propagating through the wireless channel undergoes multipath fading effect accompanied by noise and interference. Mitigation of these effects and increase in throughput is only possible if the channel is accurately estimated at the receiver in order to perform channel estimation.
\end{abstract}

Depending on slow/fast channel fading conditions, several authors suggested adaptive LMS, RLS, and NLMS based channel estimators, which either require statistical information of the channel or are not efficient enough in terms of performance or computations.

In order to overcome the above effects, the work focuses on the QR-RLS based channel estimation method for MIMO-OFDM systems. The proposed algorithm based on QR-RLS channel estimation technique provide reduce mean square error (MSE) and bit error rate (BER) compared to previous channel estimation technique.

\section{Keywords}

MIMO-OFDM System, Bit Error Rate (BER), Mean Square Error (MSE), Square Root-Recursive Least Square (QR-RLS)

\section{INTRODUCTION}

In recent years, Internet usage has increased by leaps and bounds and has billions of users. Internet usages like VOD-Video on Demand, E-Mail, Browsing, Contacts etc. demand high-speed Internet that leads to a need for broadband adoption. At the same time, cellular systems have made it possible for people to stay connected with the world from almost anywhere, resulting in a concept while On The Move. With the increase in users and their demands, the broadband market continues to grow, which in turn leads to the development of new technologies like WiMAX [1], LTE, LTE-advanced for broadband wireless. These technologies provide usage flexibility, high throughput, and more coverage.

Wireless channel [2] is the main barrier to these new technologies. It causes impairments like noise addition, interference, multipath fading effects etc. in the transmitted signal. This demands very complex algorithms in the wireless receiver to overcome these impairments. Previous technologies like GSM [3] use FDM (Frequency Division Multiplexing), while CDMA uses orthogonal codes and spread spectrum to overcome channel impairments. These systems have their own limitations. For example, FDM requires guard band for separation to overcome interference between two consecutive users. Similarly, CDMA [4] needs to generate orthogonal sequences with zero correlation which is difficult to achieve if the number of users increases indefinitely. This leads to OFDM
[5] (Orthogonal Frequency Division Multiplexing). The concept is equivalent to dividing the channel frequency response into smaller orthogonal sub-bands. Since each adjacent frequency is orthogonal to each other, it eliminates the need for guard band for separation. Simultaneously, OFDM divides high data rate signal into multiple small data rate signals. Moreover, it can be implemented with simple FFT/IFFT techniques, leading to ease of implementation.

\section{CHANNEL ESTIMATION}

Coherent demodulation acquires channel knowledge at the receiver to compensate for the channel induced distortions. The process of acquiring the channel knowledge is called channel estimation which is an integral part of most of the communication receivers nowadays.

Apart from the knowledge of channel statistics, the channel estimator also requires knowledge of the instantaneous channel values to track the channel fading and compensate for it. The available literature generally describes two types of channel estimation methods. One is based on pilot based estimation and the other is blind estimation [6]. Blind channel estimation methods avoid the use of pilots and have higher spectral efficiency. However, they suffer from high computational complexity and low convergence speed required to derive statistical information from received data. On the other hand, pilot based estimation uses pilots in block/distributed manner, depending on channel slow/fast fading conditions. The transmitter transmits multiplexed pilot symbols along with data symbols to acquire channel knowledge at the receiver. Such a channel estimation scheme is called Pilot Symbol Aided Modulation (PSAM). The estimator then uses sophisticated signal processing algorithms like LSE and MMSE to acquire the channel knowledge using pilot symbols. Several authors have proposed channel estimation in the frequency domain [7] for OFDM systems using MMSE and LSE methods. However MMSE methods require prior knowledge of channel statistics. Such information is not generally known which leads to modified LMMSE based method. Still, this method requires higher computations. On the other hand, LSE based method requires just a scalar division to estimate the channel in the frequency domain, but it leads to high channel estimation MSE error since the estimation error gets spread over the whole frequency band. This leads to time domain channel estimation which is more robust as it tracks down the inherent frequency correlation among the taps.

Literature also addresses [8] time domain channel tracking methods like MMSE, LMS, RLS adaptive estimators for OFDM systems. These methods use training symbols for estimator coefficient updating. As mentioned earlier, MMSE requires prior knowledge, while LMS method only use currently received value for filter coefficient updating, resulting in slow convergence. Filter updating based on a block of received data is more accurate, fast converging and also averages out the noise 
effect. Such methods are called RLS based channel tracking. Still, RLS based updating suffers from high computation due to matrix inversion and singularity under highly correlated channel conditions. This leads to proposed QR-RLS based channel estimation for MIMO OFDM systems, which use preamble symbols for initial channel estimation. The above method produces better results in low and moderate channel fading environments. Under fast fading channel conditions, the performance improvement is achieved by using time domain QR-RLS channel estimator in-conjunction with scattered pilots in the frequency domain.

\section{MIMO-OFDM SYSTEM}

MIMO has been created for a long time for remote frameworks. One of the most punctual MIMO to remote correspondences applications came in mid-1980 with the achievement improvements by Jack Winters and Jack Saltz of Bell Laboratories [9]. They attempted to send information from numerous clients on a similar recurrence/time channel utilizing different radio wires both at the transmitter and recipient. From that point forward, a few scholastics and architects have made noteworthy commitments in the field of MIMO. Presently MIMO innovation has stirred intrigue due to its conceivable applications in advanced TV, remote neighborhood, metropolitan zone systems and versatile correspondence. Contrasting with the Single-information single-yield (SISO) framework MIMO gives upgraded framework execution under a similar transmission condition. To begin with, MIMO framework extraordinarily builds the channel limit, which is in relative to the aggregate number of transmitter and beneficiary clusters.

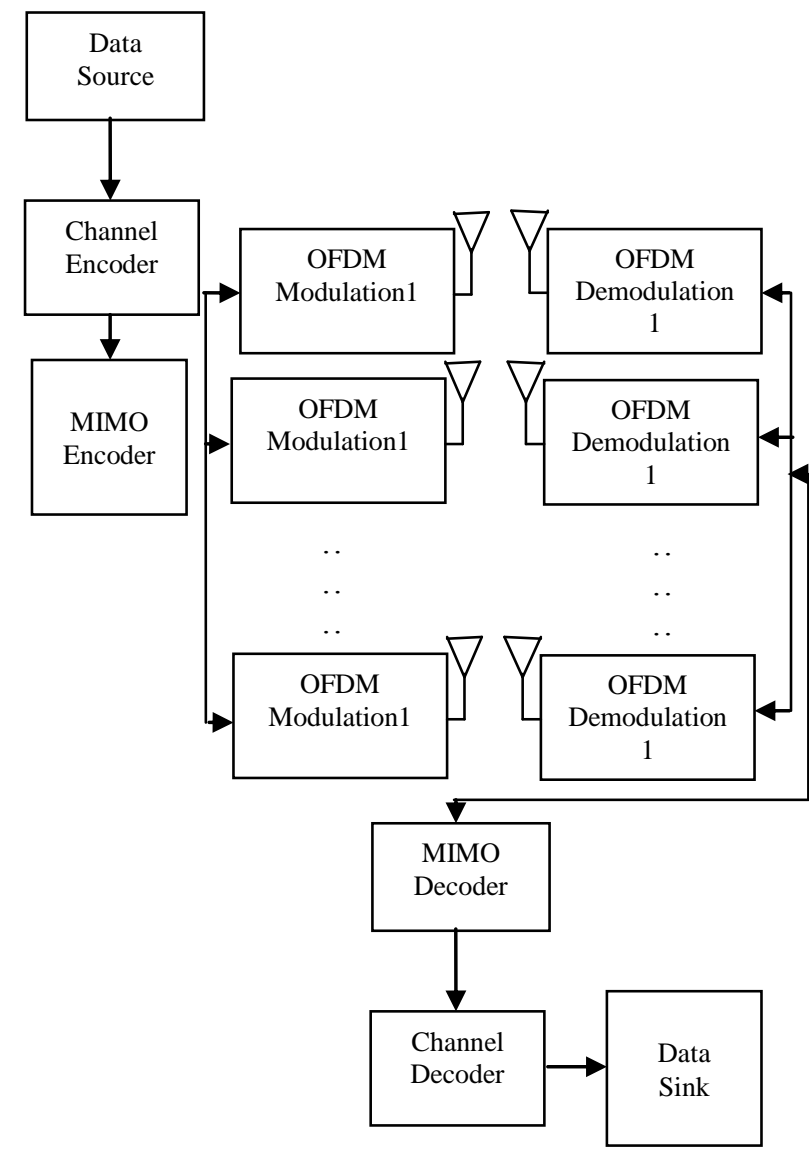

Figure 1: Block Diagram of MIMO-OFDM Communication System

\section{PROPOSED METHODOLOGY}

The MIMO-OFDM device modified into applied with the useful resource of MATLAB/SIMULINK. The execution device is binary facts this is modulated by the use of QAM and mapped into the constellation elements.

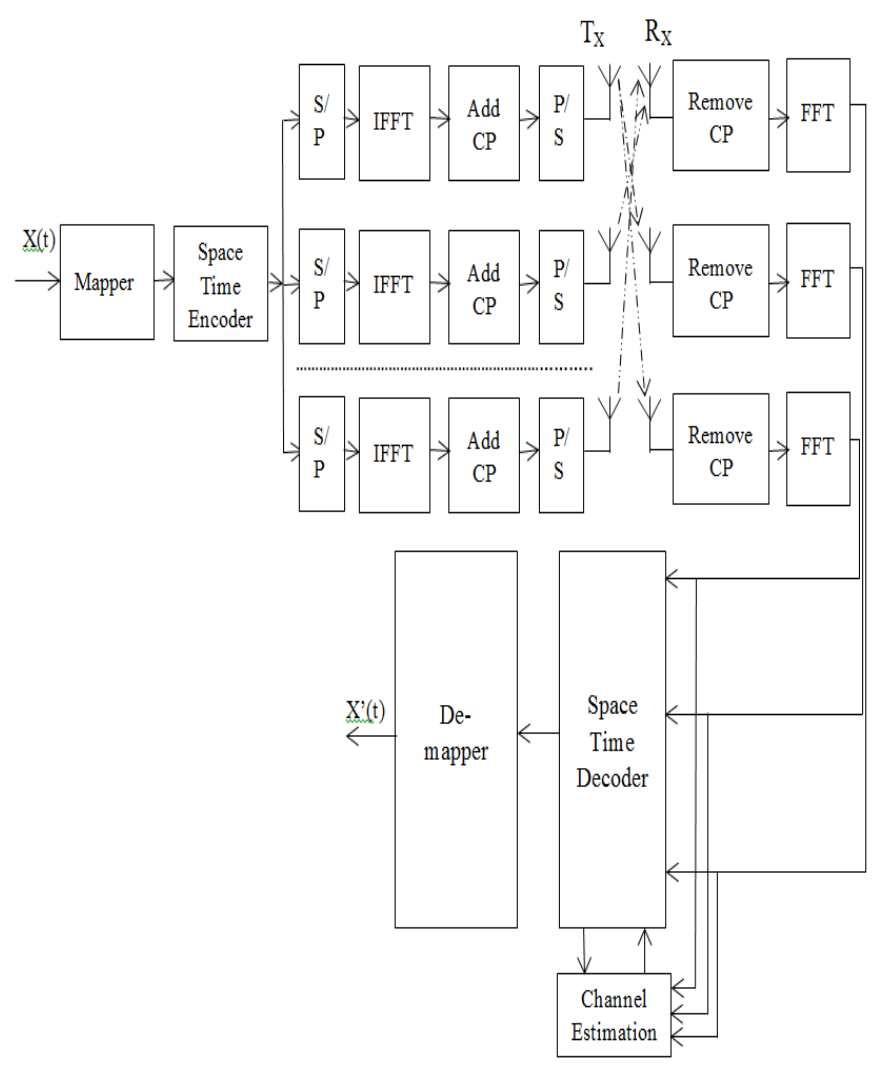

Figure 2: MIMO-OFDM System Model with Channel Estimation Technique

The virtual modulation scheme will transmit the records in parallel by means of the manner of assigning symbols to every subchannel and the modulation scheme will determine the phase mapping of sub-channels through a complex I-Q mapping vector shown in figure 2 . The complicated parallel facts stream must be converted into an analog signal this is suitable to the transmission channel.

The complicated parallel facts stream has to be transformed into an analog sign that is suitable to the transmission channel. It is performed by the cyclic prefix added to the baseband modulation signal because the baseband signal does not overlap. After that, the signal is split into the two or more part according to the requirement.

\section{SIMULATION RESULT}

In simulations, it is assumed that the system is perfectly synchronized. Different values of SNR are taken and the performance is checked.

The proposed algorithm is applied for channel estimation in MIMO OFDM system using QAM as modulation. Channel used is the Gaussian channel. Below figure shows the MSE vs SNR plot for the QR-RLS algorithm. It is seen that the curve for QRRLS indicates a decrease in BER. To begin with, the BER, overall performance isn't advanced a lot but as the SNR cost increases the BER performance also will increase. 


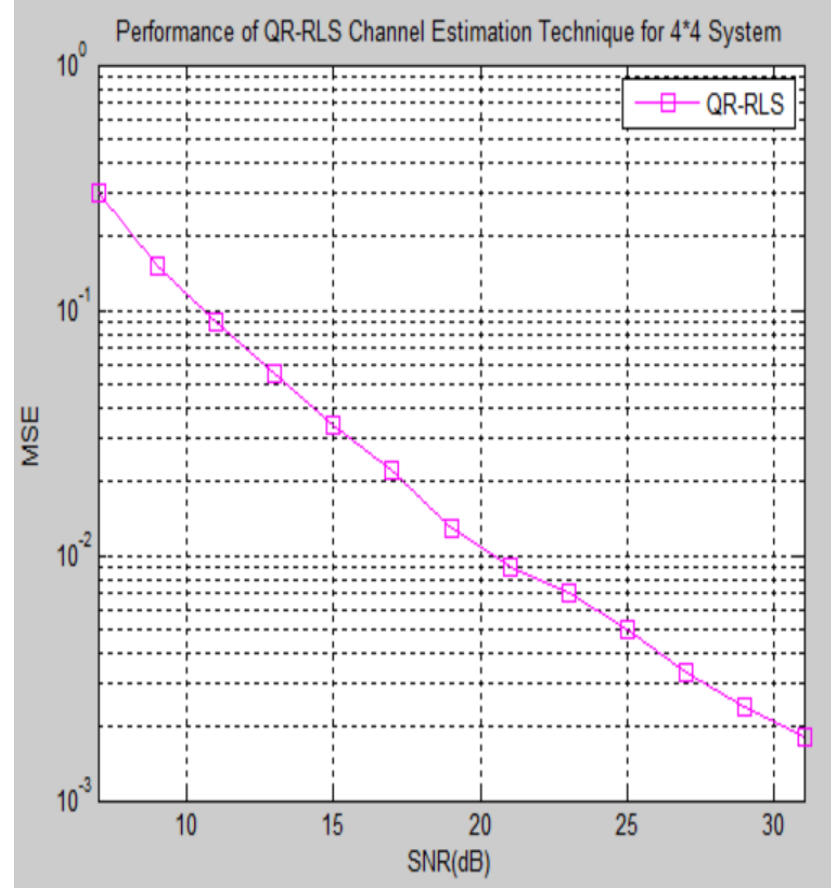

Figure 3: Performance of MSE for $4 \times 4$ MIMO-OFDM System

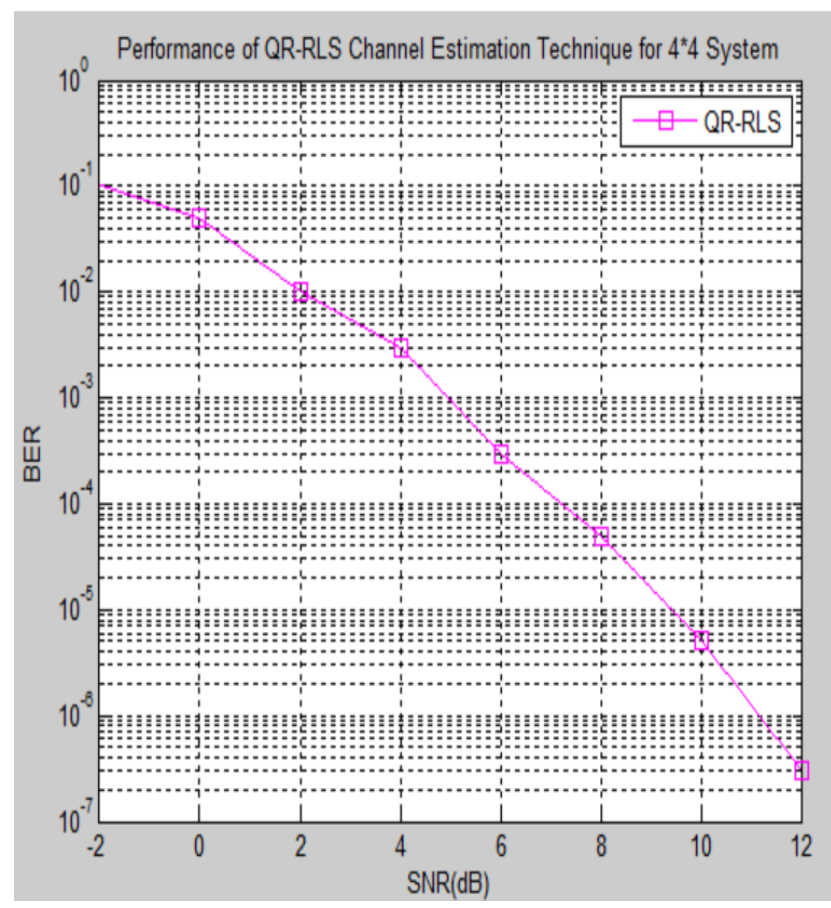

Figure 4: Performance of BER for $4 \times 4$ MIMO-OFDM System

Table 1 shows the value of bit error rate (BER) in the previous and proposed algorithm. It is clearly seen that energy/bits (SNR) increases and BER decrease in this algorithm. It is clearly seen that the QR-RLS algorithm gives the best result.
Table 1: Comparison Result for BER

\begin{tabular}{|l|l|l|}
\hline SNR (dB) & Previous Algorithm & $\begin{array}{l}\text { Proposed } \\
\text { Algorithm }\end{array}$ \\
\hline BER & 0 & 0 \\
\hline $10^{-0}$ & 1 & 0 \\
\hline $10^{-1}$ & 3.7 & 2 \\
\hline $10^{-3}$ & 6 & 4.5 \\
\hline $10^{-4}$ & 8 & 7.1 \\
\hline $10^{-5}$ & 10 & 9.2 \\
\hline $10^{-6}$ & 12 & 11.1 \\
\hline $10^{-7}$ & 14 & 13 \\
\hline
\end{tabular}

\section{CONCLUSION}

Because demands for high-rate data transmission increase very dramatically, it stimulates greater research efforts in developing wideband wireless communication systems which can support high-rate transmission over wireless channels. So the combination of MIMO and OFDM techniques (MIMO-OFDM) is an attractive method for the wireless cellular systems.

Considering accurate channel state information is essential for coherent detection and decoding in a MIMO-OFDM system, there are channel estimation methods which are proposed for the MIMO-OFDM system. The bit error rate is the main task of any wireless system. In this paper, BER, MSE, and signal to noise ratio (SNR) are maintained. The proposed algorithm is QR-RLS which maintain all the parameters in the MIMO-OFDM system. The proposed algorithm is implemented by MATLAB software and the good result is achieved compared to the existing algorithm. The proposed MIMO-OFDM system using QRRLS based channel estimation technique is also implemented in high transmitter and receiver antenna.

\section{REFERENCES}

[1] Akhilesh Venkatasubramanian, Krithika.V and Partibane. B, "Channel Estimation For A Multi-User MIMO-OFDMIDMA System", International Conference on Communication and Signal Processing, April 6-8, 2017, India.

[2] Mel Li, Xiang Wang, and Kun Zhang, "Comparative Study of Adaptive Filter Channel Estimation Technique in MIMO-OFDM System Based on STBC", Proceedings of the 2014 International Conference on Machine Learning and Cybernetics, Lanzhou, 13-16 July 2014.

[3] Biswajit Sahoo, Ravi Ranjan Prasad, and P. Samundiswary, "BER Analysis of Mobile WiMAX System using LDPC Coding and MIMO System under Rayleigh Channel", International Conference on Communication and Signal Processing, April 3-5, 2013, India.

[4] Mukesh Patidar, Rupeshdubey, Nitinkumar Jain and Saritakulpariya, "Performance Analysis of WiMAX 802.16e Physical Layer Model", 978-1-4673-1989. 8/12/\$31.00 @2012 IEEE [2]. 
[5] B. Sklar, "Digital Communications Fundamentals and Applications," Prentice Hall, Upper Saddle River, NJ, 2000.

[6] Wei Jiang and Daoben Li, "Convolutional Multi-code Multiplexing for OFDM Systems”, Beijing University of Posts and Telecommunications Beijing 100876, China (2007).

[7] Ke Chen and Xiaojing Huang, "A Novel Approach for Interference Suppression in Multi-Subband Convolutional Coded OFDM System", School of Electrical, Computer \& Telecommunications University of Wollongong, Australia (2010).

[8] Chin-Liang Wang and Shun-Sheng Wang $†$ and Hsiao-Ling Chang, "A Low-Complexity SLM Based PAPR Reduction Scheme for SFBC MIMO-OFDM Systems", 978-1-61284254-7/11/\$26.00 @2011 IEEE.

[9] Sen-Hung Wang, Student Member, IEEE, and Chih-Peng $\mathrm{Li}$, Member, IEEE, "A Low-Complexity PAPR Reduction Scheme for SFBC MIMO-OFDM Systems", IEEE
SIGNAL PROCESSING LETTERS, VOL. 16, NO. 11, November 2009.

[10] Yang Zhou and Tao Jiang, “A Novel Multi-Points Square Mapping Combined With PTS to Reduce PAPR of OFDM Signals Without Side Information", IEEE TRANSACTIONS ON BROADCASTING, VOL. 55, NO. 4, DECEMBER 2009.

[11] Yuan Jinsha, and Shi Hongmei, "A Modified Symbol Timing Synchronization Algorithm for WiMAX OFDM Systems", 2008 IEEE Pacific-Asia Workshop on Computational Intelligence and Industrial Application.

[12] J. Stott, "The DVB terrestrial (DVB-T) specification and its implementation in a practical modem," International Broadcasting Convention, pp. 255-260, 1996.

[13] ETSI, "Digital Video Broadcasting (DVB); framing structure, channel coding, and modulation for digital terrestrial television (DVB-T)," European Telecommunications Standard Institute ETSI EN 300 744, Nov 1999. 